\title{
Nonlinear Beamforming for Multiple-Antenna Assisted QPSK Wireless Systems
}

\author{
S. Chen, L. Hanzo and S. Tan \\ School of Electronics and Computer Science \\ University of Southampton, Southampton SO17 1BJ, UK \\ E-mails: $\{$ sqc,lh, st104r\}@ecs.soton.ac.uk
}

\begin{abstract}
A nonlinear beamforming aided detector is proposed for multiple-antenna assisted quadrature phase shift keying systems. By exploiting the inherent symmetry of the optimal Bayesian detection solution, a symmetric radial basis function (SRBF) detector is developed which is capable of approaching the optimal Bayesian performance using channel-impaired training data. In the uplink case, adaptive nonlinear beamforming can be implemented effectively by estimating the channel matrix based on the least squares channel estimate. Adaptive implementation of nonlinear beamforming in the downlink case by contrast is much more challenging, and we adopt a cluster-variation enhanced clustering algorithm to directly identify the SRBF centre vectors required for realising the optimal Bayesian detector.
\end{abstract}

\section{INTRODUCTION}

The ever-increasing demand for an improved throughput in wireless communication has motivated the development of adaptive antenna array assisted spatial processing techniques [1], [2], [3], [4], [5], [6], [7], including adaptive beamforming, in order to further improve the achievable spectral efficiency. Beamforming is traditionally defined as a linear processing. Upon appropriately combining the signals received by the antenna array linearly, adaptive beamforming is capable of separating user signals transmitted on the same carrier frequency, provided that the signal sources are sufficiently separated in the angular domain. Classically, this is achieved by a linear beamformer based on the minimum mean square error (LMMSE) solution [1], [4], [5], [8]. This L-MMSE beamforming design requires that the number of users supported is no more than the number of receive antenna elements. If this condition is not met, the system is referred to as rank-deficient. The optimal solution for the linear beamforming has been shown to be the minimum bit error rate (L-MBER) design [9], [10]. The L-MBER beamforming outperforms the L-MMSE one, particularly in hostile rank-deficient scenarios.

Digital communication signal detection however can be viewed as a classification problem [11], [12], [13], [14], where the receiver simply classifies the received channel-impaired signal into the most-likely transmitted symbol constellation point. For the multiple-antenna aided receiver, if one is willing to extend the beamforming process to nonlinear, substantial performance enhancement can be achieved over the linear beamforming at the cost of an increased complexity. The idea of nonlinear beamforming has been developed for binary phase shift keying systems [15], [16], where a symmetric radial basis function (SRBF) network is proposed to adaptively implement the optimal nonlinear beamforming solution. This study extends nonlinear beamforming to quadrature phase shift keying (QPSK) systems. For QPSK systems the optimal Bayesian detection solution can be expressed as a complexvalued radial basis function network [17], [18]. We further exploit the inherent symmetry of the optimal nonlinear beamforming solution and propose a SRBF network for adaptively implementing the Bayesian beamforming solution.

In Section II we present the QPSK beamforming signal model. Based on the system model of Section II, the optimal nonlinear beamforming solution is derived in Section III, where the inherent symmetric structure of the optimal Bayesian detection solution is discussed, while in Section IV the novel SRBF beamformer is presented and adaptive solutions are discussed for both uplink and downlink. For the uplink senario, adaptive nonlinear beamforming can be realised effectively by estimating the channel matrix using the least squares channel estimate (LSCE). For the downlink senario, adaptive nonlinear beamforming is proposed by adopting an enhanced $\kappa$-means clustering algorithm [16], [19]. The achievable performance of this nonlinear beamforming approach is demonstrated in Section V, and we offer our conclusions in Section VI.

\section{Multiple Antenna Assisted Beamforming}

Consider a coherent communication system that supports $M$ single-transmit-antenna users of the same carrier frequency $\omega=2 \pi f$. The receiver is equipped with a linear antenna array consisting of $L$ uniformly spaced elements, in order to achieve user separation in the angular domain [5], [7]. Assume that the channel is non-dispersive and it does not induce intersymbol interference. Then the symbol-rate complex-valued received signal samples can be expressed as [1], [4]

$$
x_{l}(k)=\sum_{i=1}^{M} A_{i} b_{i}(k) e^{j \omega t_{l}\left(\theta_{i}\right)}+n_{l}(k)=\bar{x}_{l}(k)+n_{l}(k),
$$

for $1 \leq l \leq L$, where $b_{i}(k)$ is the $k$-th symbol of user $i$, which takes values from a QPSK symbol set

$$
\begin{aligned}
& b_{i}(k) \in\left\{b^{[1]}=+1+j, b^{[2]}=-1+j,\right. \\
& \left.b^{[3]}=-1-j, b^{[4]}=+1-j\right\},
\end{aligned}
$$

$t_{l}\left(\theta_{i}\right)$ is the relative time delay at array element $l$ for source $i$, with $\theta_{i}$ being the direction of arrival for source $i, n_{l}(k)$ is the 
complex-valued Gaussian white noise with $E\left[\left|n_{l}(k)\right|^{2}\right]=2 \sigma_{n}^{2}$, and $A_{i}$ is the non-dispersive channel coefficient of user $i$. The average signal-to-noise ratio (SNR) of the system is

$$
\mathrm{SNR}=\left(\frac{1}{M} \sum_{i=1}^{M}\left|A_{i}\right|^{2}\right) \sigma_{b}^{2} / 2 \sigma_{n}^{2},
$$

where $\sigma_{b}^{2}$ is the QPSK symbol energy. Let source $i$ be the desired user and the rest of the sources be the interfering users. The desired signal-to-interferer $q$ ratio (SIR) is defined by $\operatorname{SIR}_{i, q}=\left|A_{i}\right|^{2} /\left|A_{q}\right|^{2}$, for $q \neq i$. The received signal vector $\mathbf{x}(k)=\left[x_{1}(k) x_{2}(k) \cdots x_{L}(k)\right]^{T}$ can be expressed as

$$
\mathbf{x}(k)=\mathbf{P b}(k)+\mathbf{n}(k)=\overline{\mathbf{x}}(k)+\mathbf{n}(k),
$$

where $\mathbf{P}=\left[\begin{array}{llll}A_{1} \mathbf{s}_{1} & A_{2} \mathbf{s}_{2} \cdots & A_{M} \mathbf{s}_{M}\end{array}\right]$ is the system's channel matrix with $\mathbf{s}_{i}=\left[e^{j \omega t_{1}\left(\theta_{i}\right)} e^{j \omega t_{2}\left(\theta_{i}\right)} \cdots e^{j \omega t_{L}\left(\theta_{i}\right)}\right]^{T}$ being the steering vector of source $i, \mathbf{b}(k)=\left[b_{1}(k) b_{2}(k) \cdots b_{M}(k)\right]^{T}$ and $\mathbf{n}(k)=\left[n_{1}(k) n_{2}(k) \cdots n_{L}(k)\right]^{T}$.

Traditionally, a linear beamformer is adopted to detect the desired user's signal [1], [4]. The linear beamformer for user $i$ is defined by $y_{\operatorname{Lin}}(k)=\boldsymbol{\alpha}_{i}^{H} \mathbf{x}(k)$, where $\boldsymbol{\alpha}_{i}=$ $\left[\begin{array}{llll}\alpha_{1, i} & \alpha_{2, i} & \cdots & \alpha_{L, i}\end{array}\right]^{T}$ is the complex-valued $i$-th linear beamformer's weight vector. The decision regarding the transmitted symbol $b_{i}(k)$ is given by $\hat{b}_{i}(k)=\operatorname{sgn}\left(y_{\operatorname{Lin}}(k)\right)$ with

$$
\operatorname{sgn}(y)= \begin{cases}b^{[1]}=+1+j, & y_{R} \geq 0 \text { and } y_{I} \geq 0, \\ b^{[2]}=-1+j, & y_{R}<0 \text { and } y_{I} \geq 0, \\ b^{[3]}=-1-j, & y_{R}<0 \text { and } y_{I}<0, \\ b^{[4]}=+1-j, & y_{R} \geq 0 \text { and } y_{I}<0,\end{cases}
$$

where $y_{R}=\Re[y]$ and $y_{I}=\Im[y]$ denote the real and imaginary parts of $y$, respectively. The optimal weight vector designed for the linear beamformer is known to be the L-MBER solution [9], [10]. However, we will show that the true optimal solution for the beamforming aided detector is nonlinear.

\section{Optimal Bayesian BeAmforming Solution}

Denote the $N_{b}=4^{M}$ legitimate combinations of $\mathbf{b}(k)$ as $\mathbf{b}_{q}$, $1 \leq q \leq N_{b}$. The noiseless channel output $\overline{\mathbf{x}}(k)$ takes values from the vector state set $\mathcal{X} \triangleq\left\{\overline{\mathbf{x}}_{q}=\mathbf{P b}_{q}, 1 \leq q \leq N_{b}\right\}$, and $\mathcal{X}$ can be divided into the four subsets conditioned on the values of $b_{i}(k)=b^{[m]}, 1 \leq m \leq 4$, as follows

$$
\mathcal{X}^{[m]} \triangleq\left\{\overline{\mathbf{x}}_{i}^{[m]} \in \mathcal{X}, 1 \leq i \leq N_{s b}: b_{i}(k)=b^{[m]}\right\},
$$

where the size of $\mathcal{X}^{[m]}$ is $N_{s b}=4^{M-1}$. Denote the conditional probabilities of receiving $\mathbf{x}(k)$ given $b_{i}(k)=b^{[m]}$ as $p^{[m]}(\mathbf{x}(k))=p\left(\mathbf{x}(k) \mid b_{i}(k)=b^{[m]}\right)$. According to Bayes' decision theory [20], the optimal detection strategy is

$$
\hat{b}_{i}(k)=b^{\left[m^{*}\right]}
$$

where

$$
m^{*}=\arg \max _{1 \leq m \leq 4} p^{[m]}(\mathbf{x}(k))
$$

Define the complex-valued Bayesian decision variable [18]

$$
\begin{aligned}
y_{\text {Bay }}(k) \triangleq & b^{[1]} \cdot p^{[1]}(\mathbf{x}(k))+b^{[2]} \cdot p^{[2]}(\mathbf{x}(k)) \\
& +b^{[3]} \cdot p^{[3]}(\mathbf{x}(k))+b^{[4]} \cdot p^{[4]}(\mathbf{x}(k)) .
\end{aligned}
$$

The optimal Bayesian detection rule (7) and (8) is equivalent to $\hat{b}_{i}(k)=\operatorname{sgn}\left(y_{\text {Bay }}(k)\right)$.

The conditional probability $p^{[m]}(\mathbf{x}(k))$ can be expressed as

$$
p^{[m]}(\mathbf{x}(k))=\sum_{q=1}^{N_{s b}} \beta_{q} e^{-\frac{\left\|\mathbf{x}(k)-\overline{\mathbf{x}}_{q}^{[m]}\right\|^{2}}{2 \sigma_{n}^{2}}}
$$

where $\overline{\mathbf{x}}_{q}^{[m]} \in \mathcal{X}^{[m]}$, and $\beta_{q}$ is proportional to the a priori probability of $\overline{\mathbf{x}}_{q}^{[m]}$. Since all the $\overline{\mathbf{x}}_{q}^{[m]}$ are equiprobable, $\beta_{q}=$ $\beta=\frac{1}{N_{s b}\left(2 \pi \sigma_{n}^{2}\right)^{L}}$. It can be seen that the optimal Bayesian decision variable (9) takes the structure of a complex-valued RBF network [17] with a Gaussian RBF function. The state subsets $\mathcal{X}^{[m]}, 1 \leq m \leq 4$, are distributed symmetrically with respect to each other as summarised in the following lemma. Lemma. The four subsets $\mathcal{X}^{[m]}, 1 \leq m \leq 4$, satisfy

$$
\mathcal{X}^{[2]}=+j \cdot \mathcal{X}^{[1]}, \mathcal{X}^{[3]}=-1 \cdot \mathcal{X}^{[1]}, \mathcal{X}^{[4]}=-j \cdot \mathcal{X}^{[1]} .
$$

Proof: Consider any $\overline{\mathbf{x}}_{q}^{[1]}=\mathbf{P b}_{q}^{[1]} \in \mathcal{X}^{[1]}$, where the $i$-th element of $\mathbf{b}_{q}^{[1]}$ is $b^{[1]}=+1+j$. Noting $j \cdot b^{[1]}=b^{[2]}$, $j \cdot \overline{\mathbf{x}}_{q}^{[1]}=\mathbf{P}\left(j \cdot \mathbf{b}_{q}^{[1]}\right) \in \mathcal{X}^{[2]}$. This proves the first relationship. The proofs of the other two relationships are similar. Given this symmetry, the optimal Bayesian solution (9) can alternatively be expressed as

$$
\begin{gathered}
y_{\text {Bay }}(k)=\sum_{q=1}^{N_{s b}}\left\{b^{[1]} \beta \cdot e^{-\frac{\left\|\mathbf{x}(k)-\overline{\mathbf{x}}_{q}^{[1]}\right\|^{2}}{2 \sigma_{n}^{2}}}+b^{[2]} \beta \cdot e^{-\frac{\left\|\mathbf{x}(k)-j \cdot \overline{\mathbf{x}}_{q}^{[1]}\right\|^{2}}{2 \sigma_{n}^{2}}}\right. \\
\left.+b^{[3]} \beta \cdot e^{-\frac{\left\|\mathbf{x}(k)+\overline{\mathbf{x}}_{q}^{[1]}\right\|^{2}}{2 \sigma_{n}^{2}}}+b^{[4]} \beta \cdot e^{-\frac{\left\|\mathbf{x}(k)+j \cdot \overline{\mathbf{x}}_{q}^{[1]}\right\|^{2}}{2 \sigma_{n}^{2}}}\right\},
\end{gathered}
$$

where $\overline{\mathbf{x}}_{q}^{[1]} \in \mathcal{X}^{[1]}$.

\section{Symmetric Radial Basis Function Network}

Consider how to realise the optimal beamforming solution using a radial basis function (RBF) network. The symmetry of the Bayesian solution (12) should be explicitly exploited, and we propose to use the following SRBF network for the detection of user $i$ data

$$
\begin{gathered}
y_{\mathrm{RBF}}(k)=\sum_{q=1}^{N_{c}}\left\{\alpha_{q}^{[1]} \varphi\left(\mathbf{x}(k) ; \mathbf{c}_{q}, \sigma_{q}^{2}\right)+\alpha_{q}^{[2]} \varphi\left(\mathbf{x}(k) ; j \mathbf{c}_{q}, \sigma_{q}^{2}\right)\right. \\
\left.+\alpha_{q}^{[3]} \varphi\left(\mathbf{x}(k) ;-\mathbf{c}_{q}, \sigma_{q}^{2}\right)+\alpha_{q}^{[4]} \varphi\left(\mathbf{x}(k) ;-j \mathbf{c}_{q}, \sigma_{q}^{2}\right)\right\}
\end{gathered}
$$

with the decision $\hat{b}_{i}(k)=\operatorname{sgn}\left(y_{\mathrm{RBF}}(k)\right)$, where $N_{c}$ is the number of RBF centres, $\mathbf{c}_{q}$ are the complex-valued RBF centre vectors, $\alpha_{q}^{[m]}$ for $1 \leq m \leq 4$ are complex-valued RBF weights, $\sigma_{q}^{2}$ are the RBF variances, and $\varphi(\bullet)$ is the real-valued radial basis function. In this study we adopt the Gaussian function. Since the number of users is usually known, the number of RBF centres can be set to $N_{c}=N_{s b}$. To further exploit the structure of the optimal Bayesian solution (12), the complexvalued RBF weights are set to $\alpha_{q}^{[m]}=\beta b^{[m]}, 1 \leq m \leq 4$, where $\beta>0$ is a constant. Furthermore, all the RBF variances can be set to $\sigma_{q}^{2}=\hat{\sigma}_{n}^{2}$, where $\hat{\sigma}_{n}^{2}$ is an estimate of the noise variance. Thus, adaptation of the SRBF network (13) becomes the task of finding appropriately the RBF centre vectors $\mathbf{c}_{q}$. 


\section{A. Uplink Detection}

In the uplink senario, the receiver has to detect all the users' data, and it has access to the training symbols of all the users. The most effective way of adaptive implementation of the SRBF network detector (13) is to estimate the channel matrix $\mathbf{P}$ first and then use it to calculate the state subset $\mathcal{X}^{[1]}$, which specifies the optimal Bayesian solution. Given the training data set $\{\mathbf{x}(k), \mathbf{b}(k)\}_{k=1}^{K}$, where $K$ is the number of training symbols, define the training symbol matrix and the corresponding observation matrix as $\mathbf{B}_{K}=[\mathbf{b}(1) \mathbf{b}(2) \cdots \mathbf{b}(K)]$ and $\mathbf{X}_{K}=[\mathbf{x}(1) \mathbf{x}(2) \cdots \mathbf{x}(K)]$, respectively. Then the LSCE for $\mathbf{P}$ is given by $\hat{\mathbf{P}}=\mathbf{X}_{K} \mathbf{S}_{K}^{H}\left(\mathbf{S}_{K} \mathbf{S}_{K}^{H}\right)^{-1}$. As a byproduct of the LSCE, an estimated channel noise variance $\hat{\sigma}_{n}^{2}=$ $\left\|\mathbf{X}_{K}-\hat{\mathbf{P}} \mathbf{S}_{K}\right\|^{2} / 2 K$ is also produced.

\section{B. Downlink Detection}

In the downlink senario, the task of receiver is to detect the data of the desired user $i$. During training, the receiver has the training data $\left\{\mathbf{x}(k), b_{i}(k)\right\}_{k=1}^{K}$ but it does not have access to the interfering users' data $\left\{b_{q}(k)\right\}, q \neq i$. Thus, estimating the channel matrix $\mathbf{P}$ is a challenging task. It is more feasible using the channel-impaired training data to directly adjust the SRBF network (13) and hence to approximate the optimal Bayesian solution. We adopt the enhanced $\kappa$-means clustering algorithm [16], [19] to adapt the RBF centres. Specifically, during training, the RBF centres are adjusted according to

$$
\mathbf{c}_{l}(k)=\mathbf{c}_{l}(k-1)+\mu_{c} \mathcal{M}_{l}(\check{\mathbf{x}}(k))\left(\check{\mathbf{x}}(k)-\mathbf{c}_{l}(k-1)\right),
$$

where $\mu_{c}$ is the step size,

$$
\check{\mathbf{x}}(k)= \begin{cases}+1 \cdot \mathbf{x}(k), & b_{i}(k)=b^{[1]}, \\ -j \cdot \mathbf{x}(k), & b_{i}(k)=b^{[2]}, \\ -1 \cdot \mathbf{x}(k), & b_{i}(k)=b^{[3]}, \\ +j \cdot \mathbf{x}(k), & b_{i}(k)=b^{[4]}\end{cases}
$$

and the membership function $\mathcal{M}_{l}(\mathbf{x})$ is defined as

$$
\mathcal{M}_{l}(\mathbf{x})= \begin{cases}1, & \text { if } \bar{v}_{l}\left\|\mathbf{x}-\mathbf{c}_{l}\right\|^{2} \leq \bar{v}_{q}\left\|\mathbf{x}-\mathbf{c}_{q}\right\|^{2}, \forall q \neq l, \\ 0, & \text { otherwise, }\end{cases}
$$

with $\bar{v}_{l}$ being the variation of the $l$-th cluster. The following updating rule is used to estimate the associated variation $\bar{v}_{l}$

$$
\bar{v}_{l}(k)=\mu_{v} \bar{v}_{l}(k-1)+\left(1-\mu_{v}\right) \mathcal{M}_{l}(\check{\mathbf{x}}(k))\left\|\check{\mathbf{x}}(k)-\mathbf{c}_{l}(k-1)\right\|^{2},
$$

where $\mu_{v}$ is a constant slightly less than 1.0. The initial variations $\bar{v}_{l}(0), \forall l$, are set to the same small number.

\section{Simulation Study}

A three-element antenna array was designed to support four QPSK users. Fig. 1 shows the angular positions of the four users . The simulated narrowband channels were $A_{i}=\eta_{i}(1+$ $j 0), 1 \leq i \leq 4$, where $\eta_{i}^{2}$ specified the power of user $i$. First, we demonstrated the performance improvement achievable by the optimal nonlinear beamforming over the optimal linear one. Two cases of user power distribution were considered. In the equal power (EP) case, all the four users had a same signal power. In the unequal power (UEP) case, users 1,2

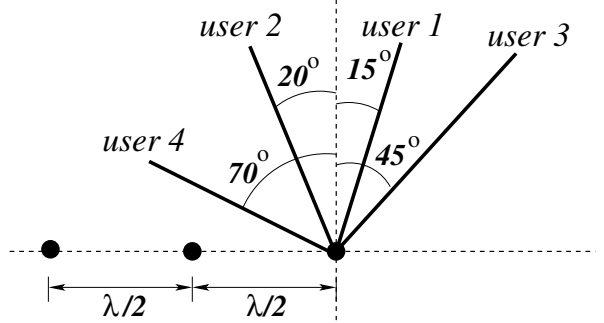

Fig. 1. Angular locations of the four QPSK users with respect to the threeelement linear antenna array having $\lambda / 2$ spacing, where $\lambda$ is the wavelength.

and 4 had a same power but user 3 had $6 \mathrm{~dB}$ more power than users 1, 2 and 4. Fig. 2 compares the BER performance of the Bayesian beamforming and the L-MBER beamforming. As expected, the Bayesian beamforming achieved much better BER performance over the optimal linear beamforming. This performance gain was of course obtained at the cost of an increased complexity. From Fig. 2 it can be seen that in the EP case the performance of the individual linear beamformer depended on the particular user's angular position as well as the other users' locations. By contrast, all the four optimal Bayesian beamformers had the similar performance. Moreover, the results of the UEP case shown in Fig. 2 also confirm that the nonlinear beamforming was much more robust to the near-far effect than the linear beamforming. Because of this remarkable robustness property, we only concentrated on the user one in the EP case when investigating adaptive implementation of the nonlinear beamforming.

The LSCE-based adaptive implementation was first investigated, and Fig. 3 depicts the user-one BER performance of the adaptive SRBF beamformer with the different numbers of training symbols $K$, given $\hat{\sigma}_{n}^{2}=\sigma_{n}^{2}$, in comparsion with the case of the perfect channel knowledge. It is seen from Fig. 3 that the LSCE-based adaptive implementation required $K=$ 64 training symbols to closely approach the optimal Bayesian performance. The performance shown in Fig. 3 was obtained by setting the RBF variance $\hat{\sigma}_{n}^{2}$ to the true noise variance $\sigma_{n}^{2}$. The influence of the RBF variance $\hat{\sigma}_{n}^{2}$ used to the BER performance of the LSCE-based adaptive SRBF beamformer is illustrated in Fig. 4, given the $\mathrm{SNR}=7 \mathrm{~dB}$ and $K=64$ training symbols. It is seen from Fig. 4 that the performance of the LSCE-based adaptive SRBF beamformer is not sensitive at all to the value of the RBF variance and there exists a large range of $\hat{\sigma}_{n}^{2}$ values which enable the LSCE-based adaptive SRBF beamformer to match the Bayesian performance.

The clustering-based adaptive SRBF beamforming was then studied. The number of the subset states was $N_{s b}=64$, and we used the first 64 data points $\check{\mathbf{x}}(k), 1 \leq k \leq 64$, as the initial RBF centres. The initial cluster variations were set to $\bar{v}_{l}(0)=0.1$ for $1 \leq l \leq N_{s b}$, and the adaptive gain for updating the cluster variations was chosen to be $\mu_{v}=0.995$. Convergence performance of the cluster-variation enhanced clustering algorithm was assessed in the simulation based on the Euclidean distance between the set of the RBF centres $\left\{\mathbf{c}_{l}\right\}_{l=1}^{N_{s b}}$ and the set of the true subset channel states $\left\{\overline{\mathbf{x}}_{l}^{[1]}\right\}_{l=1}^{N_{s b}}$. Given $\mathrm{SNR}=7 \mathrm{~dB}$, Fig. 5 plots the learning curves of the 


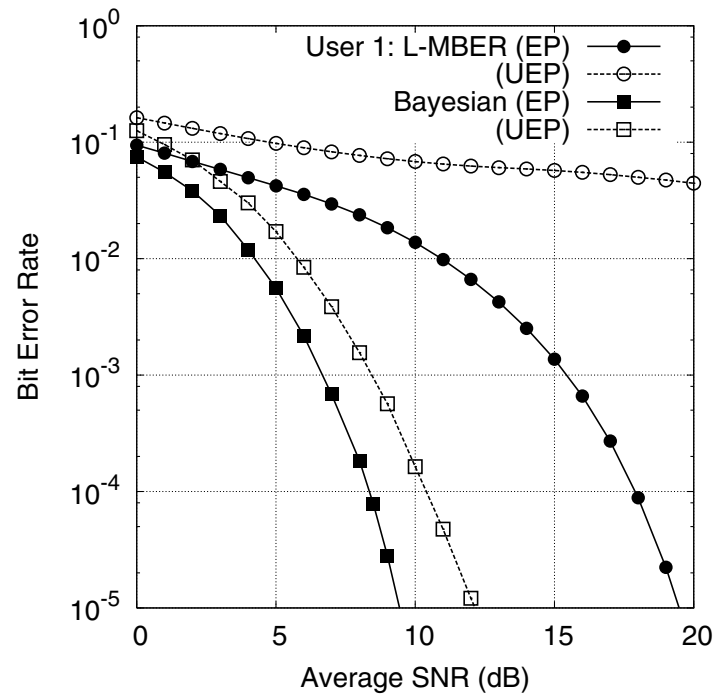

(a) user 1

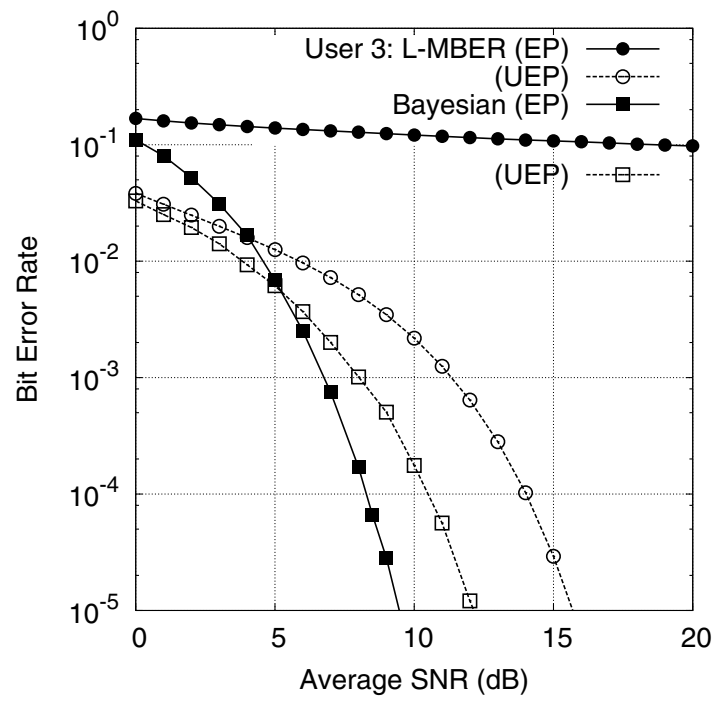

(c) user 3

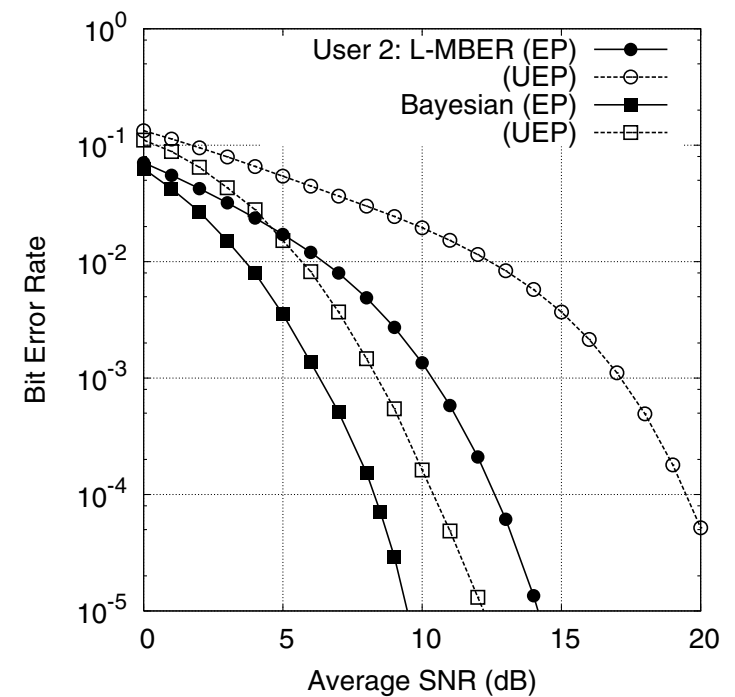

(b) user 2

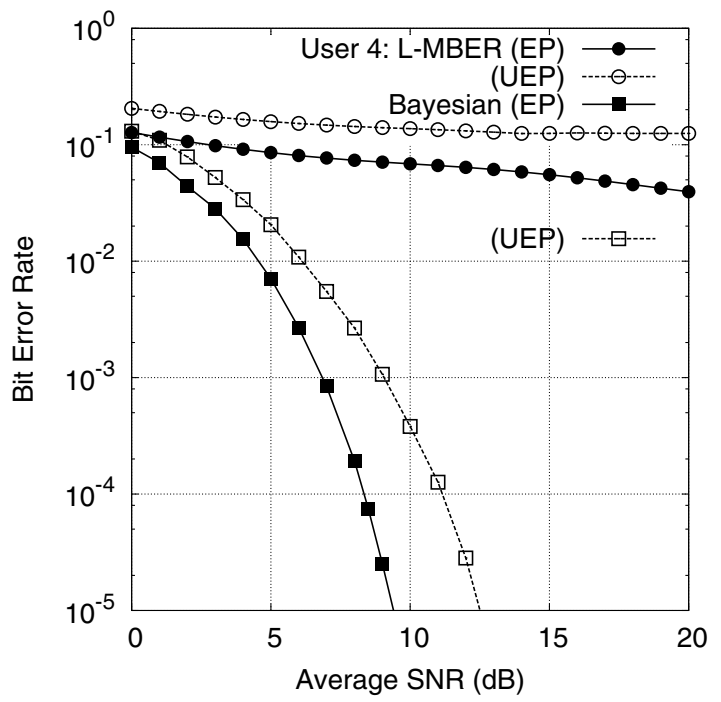

(d) user 4

Fig. 2. Bit error rate performance comparison of the optimal nonlinear beamforming with the optimal linear beamforming.

clustering algorithm averaged over ten runs for the three values of $\mu_{c}$. It is seen from Fig. 5 that for this example the best convergence performance was achieved with $\mu_{c}=0.4$. The robustness of the clustering-based adaptive SRBF beamforming with respect to the value of the RBF variance is demonstrated in Fig. 4, while Fig. 6 compares the BER performance of the clustering-based adaptive SRBF beamformer for user one after convergence with that of the optimal Bayesian beamformer, given the RBF variance $\hat{\sigma}_{n}^{2}=\sigma_{n}^{2}$.

\section{CONCLUSIONS}

A nonlinear beamforming based detector has been extended to multiple-antenna assisted QPSK wireless systems. It has been demonstrated that nonlinear beamforming is capable of substantially improving the achievable system performance and significantly increasing user capacity over the traditional linear beamforming, at the cost of an increased computational complexity. By exploiting the inherent symmetry of the optimal Bayesian solution, a novel SRBF network has been proposed for adaptive nonlinear beamforming. In the uplink senario, adaptive SRBF beamforming has been implemented efficiently by estimating the system's channel matrix based on the LSCE. For the more challenging downlink senario, the cluster-variation enhanced clustering algorithm has been adopted to implement the adaptive SRBF beamforming. The robustness of the adaptive SRBF beamformer with respect to the $\mathrm{RBF}$ variance used has been verified in the simulation.

\section{REFERENCES}

[1] J. Litva and T.K.Y. Lo, Digital Beamforming in Wireless Communications. London: Artech House, 1996.

[2] J.H. Winters, "Smart antennas for wireless systems," IEEE Personal Communications, vol.5, no.1, pp.23-27, 1998.

[3] P. Vandenameele, L. van Der Perre and M. Engels, Space Division Multiple Access for Wireless Local Area Networks. Boston: Kluwer Academic Publishers, 2001. 


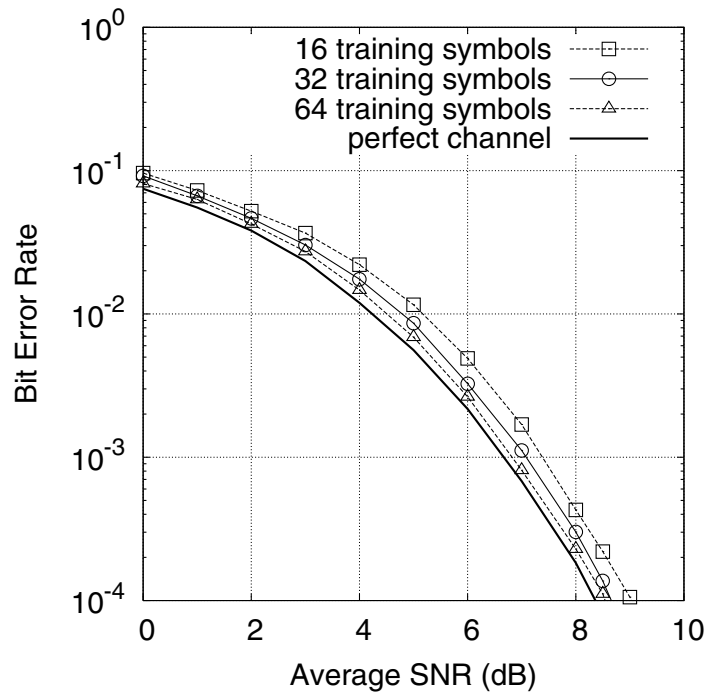

Fig. 3. User-one bit error rate performance of the LSCE-based adaptive SRBF beamformer for the equal power case, given $\hat{\sigma}_{n}^{2}=\sigma_{n}^{2}$.

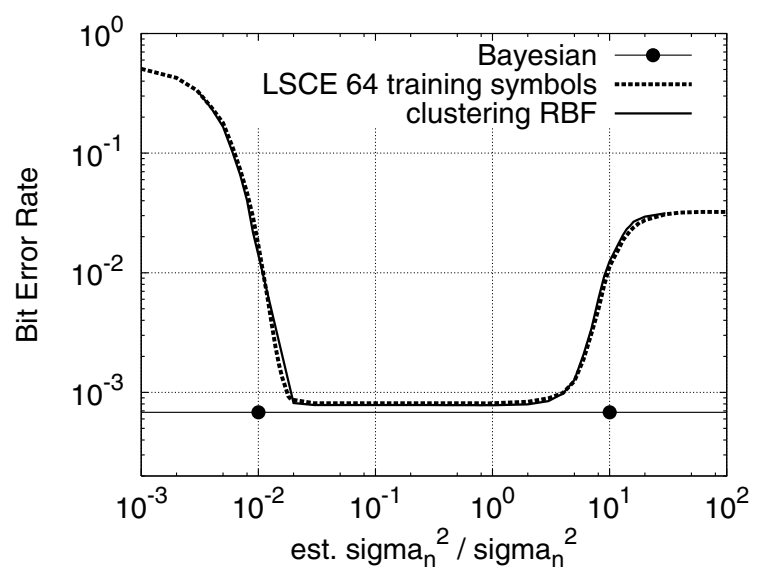

Fig. 4. The influence of the RBF variance on the bit error rate performance of the LSCE-based and clustering-based adaptive SRBF beamformers for user one of the equal power case, given $\mathrm{SNR}=7 \mathrm{~dB}$.

[4] J.S. Blogh and L. Hanzo, Third Generation Systems and Intelligent Wireless Networking - Smart Antennas and Adaptive Modulation. Chichester, U.K.: Wiley, 2002.

[5] A. Paulraj, R. Nabar and D. Gore, Introduction to Space-Time Wireless Communications. Cambridge, U.K.: Cambridge University Press, 2003.

[6] A.J. Paulraj, D.A. Gore, R.U. Nabar and H. Bölcskei, "An overview of MIMO communications - A key to gigabit wireless," Proc. IEEE, vol.92, no.2, pp.198-218, 2004.

[7] D. Tse, and P. Viswanath, Fundamentals of Wireless Communication. Cambridge, U.K.: Cambridge University Press, 2005.

[8] D.N.C. Tse and S.V. Hanly, "Linear multiuser receivers: effective interference, effective bandwidth and user capacity," IEEE Trans. Information Theory, vol.45, no.2, pp.641-657, 1999.

[9] S. Chen, N.N. Ahmad and L. Hanzo, "Adaptive minimum bit error rate beamforming," IEEE Trans. Wireless Communications, vol.4, no.2, pp.341-348, 2005.

[10] S. Chen, L. Hanzo, N.N. Ahmad and A. Wolfgang, "Adaptive minimum bit error rate beamforming assisted receiver for QPSK wireless communication," Digital Signal Processing, vol.15, no.6, pp.545-567, 2005.

[11] K. Abend and B.D. Fritchman, "Statistical detection for communication channels with intersymbol interference," Proc. IEEE, vol.58, no.5, pp.779-785, 1970.

[12] S. Chen and B. Mulgrew, "Overcoming co-channel interference using an adaptive radial basis function equaliser," Signal Processing, vol.28,

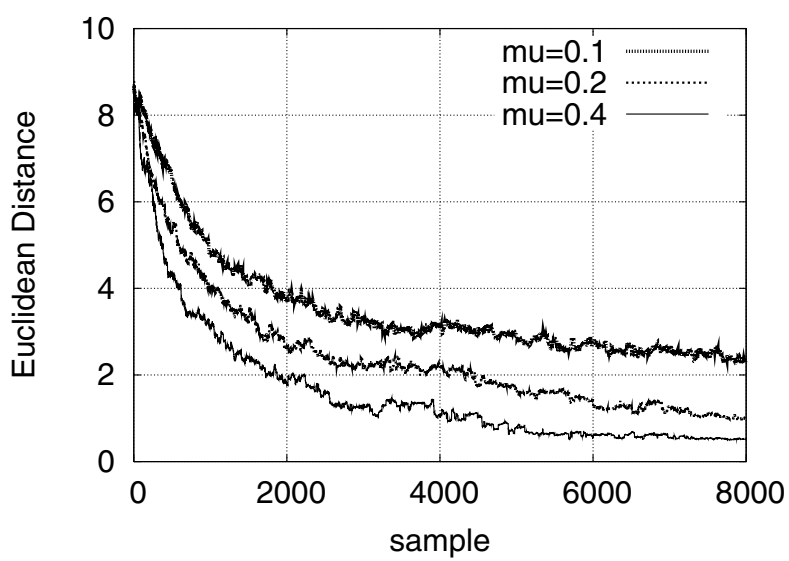

Fig. 5. Learning curves of the cluster algorithm for user one of the equal power case, in terms of Euclidean distance between the RBF centres and true channel states averaged over ten runs, given $\mathrm{SNR}=7 \mathrm{~dB}$.

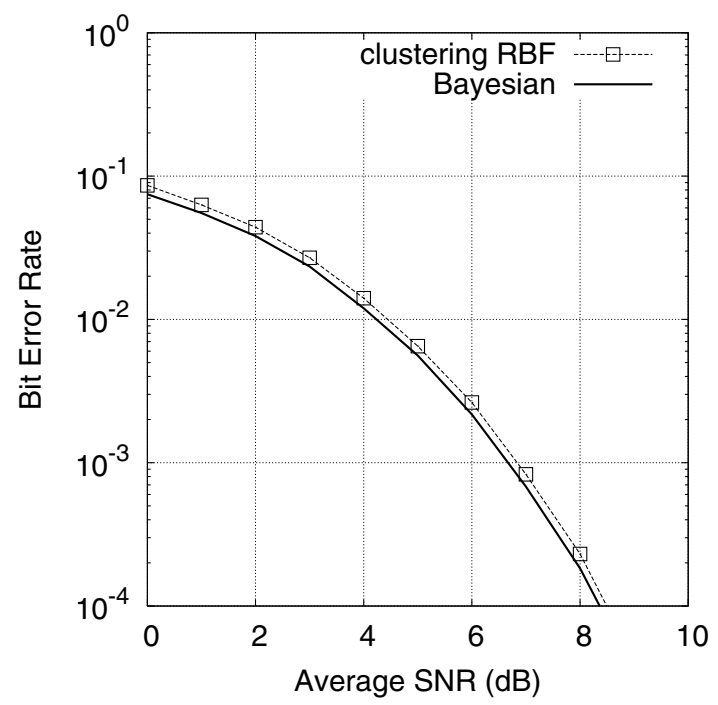

Fig. 6. User-one bit error rate performance of the clustering-based adaptive SRBF beamformer for the equal power case, given $\hat{\sigma}_{n}^{2}=\sigma_{n}^{2}$, in comparison with the optimal Bayesian beamforming performance.

no.1, pp.91-107, 1992.

[13] S. Chen, S. McLaughlin, B. Mulgrew and P.M. Grant, "Adaptive Bayesian decision feedback equaliser for dispersive mobile radio channels," IEEE Trans. Communications, vol.43, no.5, pp.1937-1946, 1995.

[14] S. Chen, A.K. Samingan and L. Hanzo, "Support vector machine multiuser receiver for DS-CDMA signals in multipath channels," IEEE Trans. Neural Networks, vol.12, no.3, pp.604-611, 2001.

[15] S. Chen, K. Labib, R. Kang and L. Hanzo, "Adaptive radial basis function detector for beamforming," in Proc. ICC 2007 (Glasgow, Scotland), June 24-28, 2007, pp.2967-2972.

[16] S. Chen, K. Labib and L. Hanzo, "Clustering-based symmetric radial basis function beamforming," IEEE Signal Processing Letters, vol.14, no.9, pp.589-592, 2007.

[17] S. Chen, S. McLaughlin and B. Mulgrew, "Complex-valued radial basis function network, Part I: Network architecture and learning algorithms," Signal Processing, vol.35, pp.19-31, 1994.

[18] S. Chen, S. McLaughlin and B. Mulgrew, "Complex-valued radial basis function network, Part II: Application to digital communications channel equalisation," Signal Processing, vol.36, pp.175-188, 1994.

[19] C. Chinrungrueng and C.H. Séquin, "Optimal adaptive $\kappa$-means algorithm with dynamic adjustment of learning rate," IEEE Trans. Neural Networks, vol.6, no.1, pp.1873-1896, 1995.

[20] R.O. Duda and P.E. Hart, Pattern Classification and Scene Analysis. New York: Wiley, 1973. 